\title{
Non-Newtonian and flow pulsatility effects in simulation models of a stented intracranial aneurysm
}

\author{
M. Cavazzuti ${ }^{\mathrm{a}}$, M.A. Atherton ${ }^{\mathrm{b}, *}$, M.W. Collins ${ }^{\mathrm{b}}$, G.S. Barozzi ${ }^{\mathrm{a}}$ \\ ${ }^{a}$ Dipartimento di Ingegneria Meccanica e Civile, Università degli Studi \\ di Modena e Reggio Emilia, via Vignolese 905, 41125 Modena, Italy \\ ${ }^{b}$ School of Engineering and Design, Brunel University, West London, UB8 3PH, UK
}

\begin{abstract}
Three models of different stent designs implanted in a cerebral aneurysm, originating from the Virtual Intracranial Stenting Challenge 2007 (VISC'07), are meshed and the flow characteristics simulated using commercial Computational Fluid Dynamics (CFD) software in order to investigate the effects of non-Newtonian viscosity and pulsatile flow. Conventional mass inflow and Wall Shear Stress (WSs) output are used as a means of comparing the CFD simulations. In addition, a WSS distribution is presented, which clearly discriminates in favour of the stent design identified by other groups. We conclude that non-Newtonian and pulsatile effects are important to include in order to avoid underestimating wss, understand dynamic flow effects and to discriminate more effectively between stent designs.
\end{abstract}

Keywords: cerebral aneurysm, stent, VISC'07, wall shear stress distribution, pulsatile flow

\footnotetext{
*corresponding author

Email address: mark.atherton@brunel.ac.uk; +44(0)1895 274000 (M.A. Atherton)
}

Preprint submitted to Proc. IMechE, Part H: Journal of Engineering in MedicineOctober 1, 2010 


\section{Introduction}

The Virtual Intracranial Stenting Challenge (VISC 2007), uses benchmark models to assess the effectiveness of state-of-the-art numerical modelling of blood flow in stented cerebral aneurysms (Radaelli et al. 2008). This international initiative provides a means of comparing new research with the extensive work on aneurysm haemodynamic effects of stents reported in the literature. In particular, an emphasis on considering patient-specific models to assess stent designs has meant that differences in processing geometry and generating meshes has made results difficult to replicate. VISC'07 enabled differences caused by grid generation and CFD implementation to be analysed via standardised geometry, stent deployment and CFD boundary conditions. Despite the VISC'07 computational model assuming Newtonian viscosity and steady-state conditions, Radaelli et al. highlighted the desirability of considering the effects of non-Newtonian viscosity and flow pulsatility in future work for purposes of clinical relevance. This desirability is evident in the light of other recent computational aneurysm studies.

Newtonian viscosity has been widely assumed, with Utter and Rossman 2007 referring back to Perktold et al. 1989, Balossino et al. 2008 omitting any mention of it, Karmonik et al. 2008 (admittedly in extended abstract form) not giving a justification and, recently, Shimogonya et al. 2009 citing the views of Steinman et al. 2003 and Ishikawa et al. 1998 that non-Newtonian rheology only has second-order effects. Fu et al. 2010 also omitted the nonNewtonian character of blood in their numerical comparison of five stent 
models in the same internal carotid aneurysm used in VISC'07, and in this paper. Their assumption was supported by previous findings by Cebral et al. 2005 who had compared Newtonian and non-Newtonian fluids on the same geometry, and concluded that the rheological model adopted did not affect substantially the main flow features. Stent performances were compared at steady-state by $\mathrm{Fu}$ et al. 2010. In that case, however, the simplification was adopted for computational, rather than physical, reasons. Pulsatility is included in the papers by Utter and Rossman 2007, Karmonik et al. 2008, Shimogonya et al. 2009 and Fu et al. 2010.

Accommodating non-Newtonian viscosity and pulsatility, therefore, could both check the former's previously assumed lack of significance and confirm the latter's importance, which was briefly outlined in a recent report (Cavazzuti et al. 2010). The importance of flow pulsatility had actually been considered minor by Kim et al. 2008, while performing a comparison study of the steady-state and pulsatile flow in an aneurismal cavity. They found that the main flow features (vortex patterns and velocity distributions) were essentially the same at three selected time points in the pulse cycle, even if differences were noted in the velocity and pressure magnitudes. In contrast, Valencia et al. 2006 investigated the effects of aneurysm geometry and non-Newtonian blood properties on the flow characteristics, wall pressure and wall shear stress, for a basilar artery, on account of a pulsatile load. Two models representative of a terminal aneurysm of the basilar artery were investigated, either adopting the Newtonian or the Herschel-Bulkley nonNewtonian rheological model. Results were also compared with predictions 
for a healthy basilar artery. A main finding was that the influence of the rheological model (Newtonian or non-Newtonian) is highly sensitive to the aneurysm geometry, in particular when a free jet is created at the sac inlet. Newtonian jets were actually found to be much less stable that their nonNewtonian counterparts, giving rise to oscillatory flows along the pulse cycle. Even if no stent analysis was included in the study, the work of Valencia et al. 2006 is of great importance in the present context, since it emphasizes that the pulsatile and non-Newtonian characters of blood should both be considered in the analysis of saccular aneurysms. As far as the choice of the most appropriate non-Newtonian model is concerned, recent work due to Fisher and Rossman 2006 compares four alternative non-Newtonian models, including the Casson, the generalized power law, and two forms of the Carreau model, all with appropriate values of the constants so as to fit the rheological properties of blood. A range of four different aneurysm morphologies was explored. The main conclusions were that: i) in spite of the huge amount of computational work performed, the importance of non-Newtonian effects is not evident, ii) different rheological models give different results, but, overall, the morphological aspects of the aneurysm are more influential than the choice of the rheological model on results such as Wss distribution, iii) such effects are more pronounced within aneurysms than in the parent vessels, and, iv) non-Newtonian effects are more influential at bifurcations than for sidewall aneurysms. The latter conclusion tends to validate the observations of Valencia et al. 2006. Finally, the importance of pulsatility in the prediction of aneurysm formation is stressed further by the recent proposal 
of a novel haemodynamic index, designated Gradient Oscillatory Number (GON), based on cycle-averaged spatial gradients of wss (Shimogonya et al. 2009).

Cerebral stents are one of several treatment options for restoring healthy blood flow through a cranial artery with a sac-like dilated wall at risk of rupture, known as an Intracranial Aneurysm (IA). Stents have often been used as an assist device in the deployment of coils. However, when employed as a flow diverter in an IA, a stent serves to occlude the entrance thus diverting flow away from the aneurysm. An additional effect of the stent mesh design is that its porosity can encourage a coagulating flow condition within the aneurysm that will stabilise the fragile sac and isolate it from the parent artery flow. This means that the blood flow characteristics through the stent wall to the aneurysm need to be markedly different to those close to the artery wall within the stent tube, and so by association, the stent design should ideally promote these dual effects. This is a requirement beyond those for stents generally, where the focus is on minimal disturbance to the arterial flow (Atherton et al. 2002, Balossino et al. 2008).

Wall Shear Stress (Wss) is the measure commonly used in Computational Fluid Dynamics (CFD) to indicate the frictional interaction between fluid and wall. In clinical terms, it has been postulated since 1971 (Caro et al. 1971) that atheroma of general arteries are prone to occur in regions of low wall shear stress. This predisposition is increased when the flow is oscillatory and most intimal thickening is found in regions where the average wss is less than $1 \mathrm{~Pa}$. Artery cells have been observed to be very sensitive to the mechanical 
stress generated by blood flow, in particular Wss (Punchard et al. 2007). Endothelial cells appear to move away from abrupt changes in wall shear stress causing a thinning of the vessel wall. Generally for an artery, thrombi formation in small regions of low wss will be inhibited by the increased amounts of nitric oxide produced in the surrounding dominant regions of higher wss (greater than 1.5 Pa). In contrast, CFD and experimental studies (Liou and Li 2008) of the flow inside the sac of an aneurysm show that it can be dominated by regions of low wss, which when below the critical value of 0.5 $\mathrm{Pa}$ produces an environment of extreme thrombus formation. Other CFD studies (Utter and Rossman 2007, Boussel et al. 1971) report that low Wss at the aneurysm fundus can trigger a biological response leading to rupture and hence to internal haemorrhage. These apparently contradictory statements do highlight that clear information on the wss values across regions of the aneurysm are important in assessing the effect of a stent design. We follow the ethos of VISC'07 in that the clinical significance of WSS is not the focus of our work, rather to assess how it is influenced by stent design, arterial geometry and flow conditions on account of the non-Newtonian character of blood, which has been demonstrated to have a definite effect on wss and distribution in saccular aneuryms, and the pulsatile nature of flow.

\section{Methods}

\subsection{Simulation input}

The models provided by VISC'07 in Stereolithography (STL) format included: an anatomical model of an unstented cerebral artery with an aneurysm, 
and three stent models for coupling with three anatomical models (Figure 1) in which the unstented anatomical model was slightly modified in order to account for the deformation of the artery caused by the stent implantation.

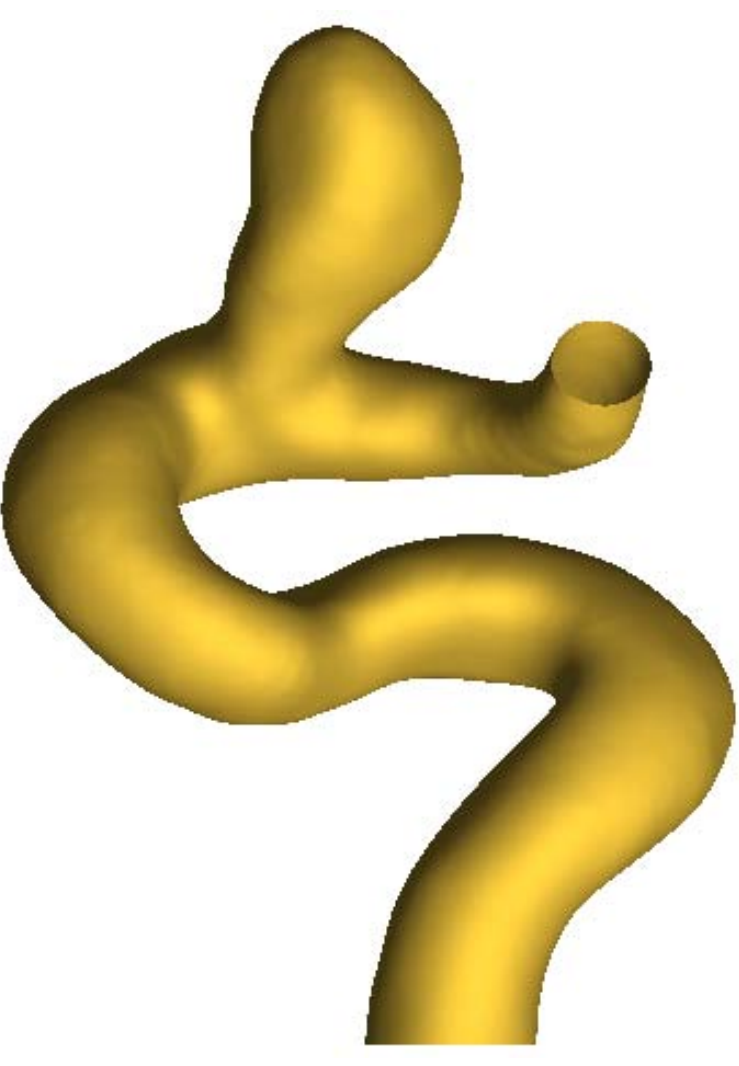

(a) Unstented aneurysm

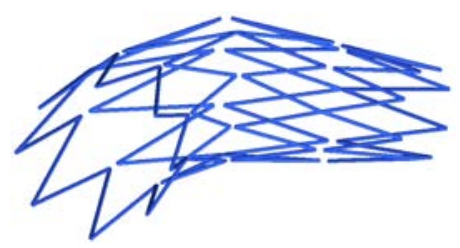

(b) Stent 1

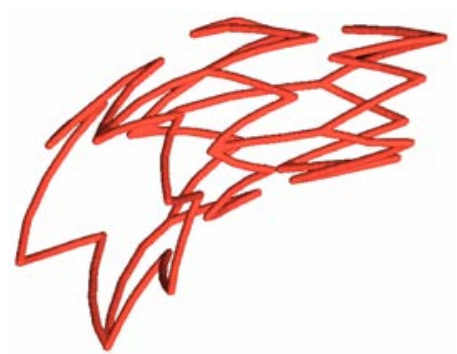

(c) Stent 2

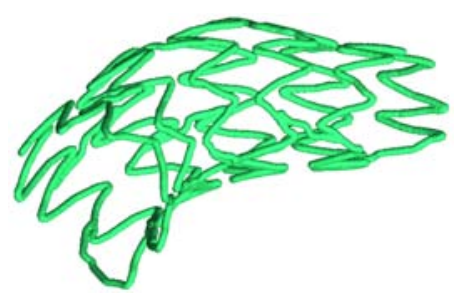

(d) Stent 3

Figure 1: Unstented aneurysm and the three VISC'07 stent models

The STL file essentially consisted of an open surface mesh, which in the case of the artery and the aneurysm duct was 59888 triangular surface elements and 30012 nodes, with finer surface meshing of the stent struts.

The fluid region of interest for the CFD simulations was obtained us- 
ing Magics software (from Materialise, Belgium) to perform the necessary Boolean operations on the surface mesh models and remove intersecting and overlapping triangles, and also close the open inlet and outlet sections of the artery model.

\subsection{Volume meshing}

In order to assess the mesh independence of the model, the volume mesh size was set to obtain meshes with approximately 1.15 million, 2.30 million and 4.60 million tetrahedral elements for the whole domain. Overall, ten different volume meshes were created: three meshes as described above for each of the three stented arteries plus one of approximately 1.35 million elements for the unstented artery.

The third stented artery had convergence problems with the 1.15 million and the 4.60 million element meshes. Therefore a grid adaption process was performed within the CFD solver on these meshes such that each tetrahedral element touching the wall was split into 8 tetrahedral elements; increasing the meshes from 1.15 to 2.25 million elements and from 4.60 to 8.35 million elements, respectively. This meant that while CPU time increased, it was possible to reach a good convergence in every CFD simulation.

The volume meshes were read into Gambit (ANSYS), where the surface of the model was split into inlet, outlet and wall and the mesh was then exported into Fluent (ANSYS) CFD code. 


\subsection{Simulation method and settings}

The following boundary conditions were used as set by VISC'07: uniform velocity with $2.36 \frac{\mathrm{g}}{\mathrm{s}}$ mass flow rate at the inlet, zero gauge pressure on the outlet and no-slip walls.

A steady-state laminar solver was used with second order upwind momentum discretization and SIMPLE pressure-velocity coupling. The arterial fluid was as blood with the following properties: density $(\rho) 1060 \frac{\mathrm{kg}}{\mathrm{m}^{3}}$ and viscosity $(\mu) 4 \mathrm{cP}$.

A Newtonian model using a constant viscosity formulation can underestimate the WSS in a CFD analysis at lower velocity gradients (Chen et al. 2006, Lee and Steinman 2006, Gijsen et al. 1999a,b) and so a non-Newtonian formulation was also investigated, using the Fluent power-law option for dynamic viscosity (Fluent 2006):

$$
\mu_{\min }<\mu=k \cdot \dot{\gamma}^{n-1}<\mu_{\max }
$$

where $\mu$ is the dynamic viscosity in $\frac{\mathrm{kg}}{\mathrm{ms}}, k$ is the consistency index whose value is $0.0161 \frac{\mathrm{kgs}^{n-2}}{\mathrm{~m}}, n$ the power-law index is 0.63 , and $\dot{\gamma}$ is the shear rate in $\mathrm{s}^{-1}$ (Owen et al. 2005). $\mu_{\min }$ and $\mu_{\max }$ are lower and upper limits of the power-law function being set to $10^{-5} \frac{\mathrm{kg}}{\mathrm{m} \mathrm{s}}$ and $1 \frac{\mathrm{kg}}{\mathrm{ms}}$ respectively. The Fluent power-law option for dynamic viscosity (Fluent 2006) is a valid option at lower shear rates (Johnson et al. 2004, Shibeshi and Collins 2005) and was therefore preferred to the more sophisticated, but more complex and computationally demanding, Casson and Carreau alternative models (see Fisher and Rossman 2006). 
Pulsatility was addressed through unsteady simulations configured for the unstented and the three stented cases. A heart beat rate of 70 beats per minute was represented using: the mass flow rate at the inlet, as specified by VISC'07; and a wave function whose average was set to $2.36 \frac{\mathrm{g}}{\mathrm{s}}$ with a uniform velocity profile of $0.179 \frac{\mathrm{m}}{\mathrm{s}}$.

Sections were traced along the artery from the inlet to the outlet and along the aneurysm at roughly $4 \mathrm{~mm}$ intervals, for Fluent post-processing purposes (Figure 2). These sections cut the artery and the aneurysm into several segments. Overall, 11 sections were traced (plus the inlet and the outlet sections), dividing the artery and the aneurysm into 12 segments. Three sections (numbers 9, 10 and 11) and three segments (numbers X, XI and XII) were located in the aneurysm region.

Section 9 corresponds to the aneurysm inlet since it is traced approximately over the minimum section area of the aneurysm and is comparable with that of cut-plane P2 in Radaelli et al. 2008. Sections 10 and 11 are, respectively, roughly $\frac{1}{3}$ and $\frac{2}{3}$ along the aneurysm height.

\subsection{WSS distribution}

The aim of implanting a stent in a cerebral aneurysm is to restore a healthy blood flow, that is, to induce the blood flow to remain inside the artery and not to enter the aneurysm region. A lower quantity of blood entering the aneurysm will produce a lower blood velocity inside the aneurysm, which itself results in lower wss. Moreover, low mass flow rate and low wsS encourages a coagulating blood flow condition within the aneurysm. 


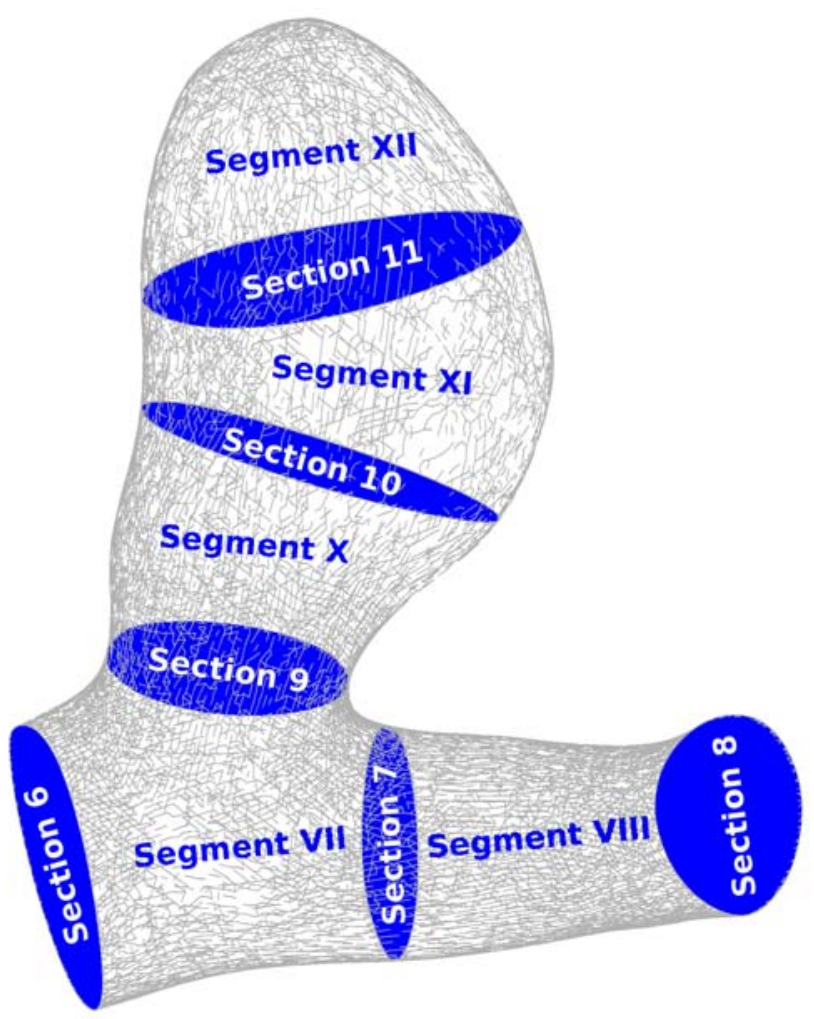

Figure 2: Sections of aneurysm model for post processing

In post-processing the CFD simulations the maximum or average wSS in Segments VII, X, XI and XII could be considered. However, as the CFD meshing is different from case to case then the maximum quantities are not particularly meaningful since they are easily affected by discretization. Beyond consideration of the maximum and average WSs, the authors believe that WSS distribution inside the aneurysm provides an indication of the patient risk that requires clinical interpretation.

This Wss distribution is determined by calculating the area of the aneurysm 
where the value of WSs is within defined intervals of $0.1 \mathrm{~Pa}$ width, starting from $0.0 \mathrm{~Pa}$ up to $3.0 \mathrm{~Pa}$. Where wss is above $3.0 \mathrm{~Pa}$, the areas are summed. These data are then used to determine the wss distribution and the cumulative distribution in each segment of the aneurysm.

\section{Results}

\subsection{Volume mesh size sensitivity}

A grid independency procedure on the stented aneurysm models, using three tetrahedral volume meshes, identifies a suitable meshing. Table 1 compares the mass flow through Section 9 for mesh sizes of 1.15, 2.30 and 4.60 million elements in Newtonian cases. It can be noted that the largest error is found in Stent 3 and that is where some convergence problems were encountered. From this comparison it was decided to continue the study using the 2.30 million elements meshes.

Table 1: Mesh sensitivity comparison based on mass flow through Section 9

\begin{tabular}{|c|c|c|c|c|}
\cline { 2 - 5 } \multicolumn{1}{c|}{} & \multicolumn{4}{c|}{ Mass Flow through Section 9 (g/s) } \\
\cline { 2 - 5 } \multicolumn{1}{c|}{} & Mesh 1.15M & Mesh 2.30M & Mesh 4.60M & max diff. \\
\hline Stent 1 & 0.3370 & 0.3391 & 0.3391 & $0.62 \%$ \\
\hline Stent 2 & 0.3414 & 0.3427 & 0.3402 & $0.73 \%$ \\
\hline Stent 3 & 0.2652 & 0.2738 & 0.2618 & $4.58 \%$ \\
\hline
\end{tabular}




\subsection{Maximum wss location}

In all the simulations an area was found in Segment X, adjacent to Section 9, where the wss is much higher than any other place in the aneurysm region (up to $18 \mathrm{~Pa}$ ). This region coincides with where the blood flow impinges on the wall when entering the aneurysm (Figure 3). Even if the wss is high in this region, it is still near to the main artery stream where the wall of the artery is not particularly damaged (thinned).

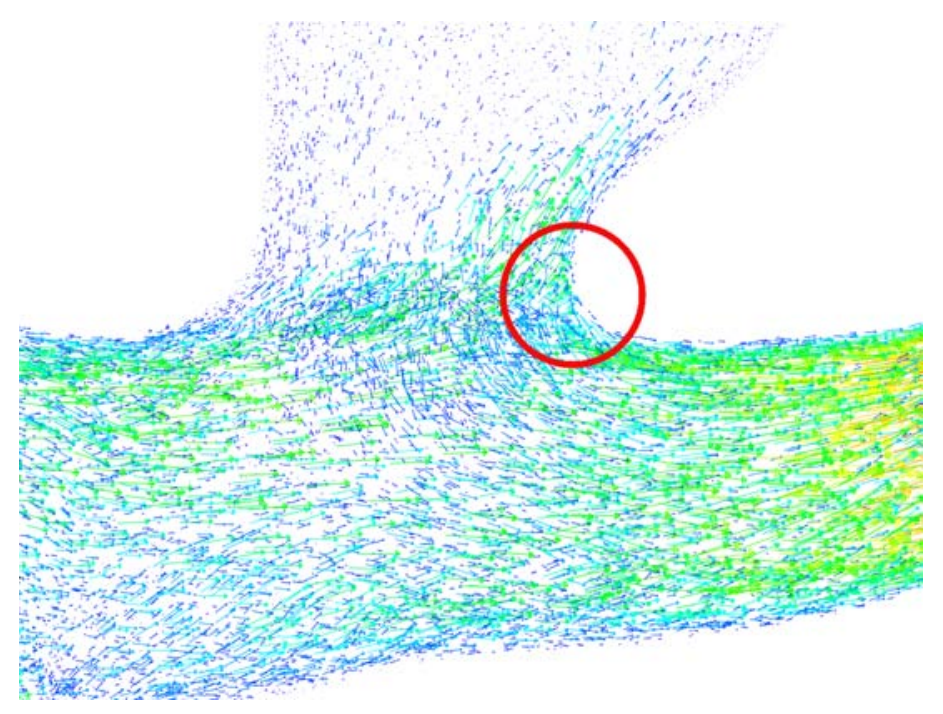

Figure 3: Velocity vectors show where flow impinges on aneurysm neck through Stent 3

\subsection{Newtonian vs. non-Newtonian}

\subsubsection{Average mass flow rate}

Considering the reductions in mass flow rates through the three sections based on the non-Newtonian blood model (see Table 2), Stent 1 and Stent 2 are comparable. Stent 2 has the highest mass flow rate through Section 


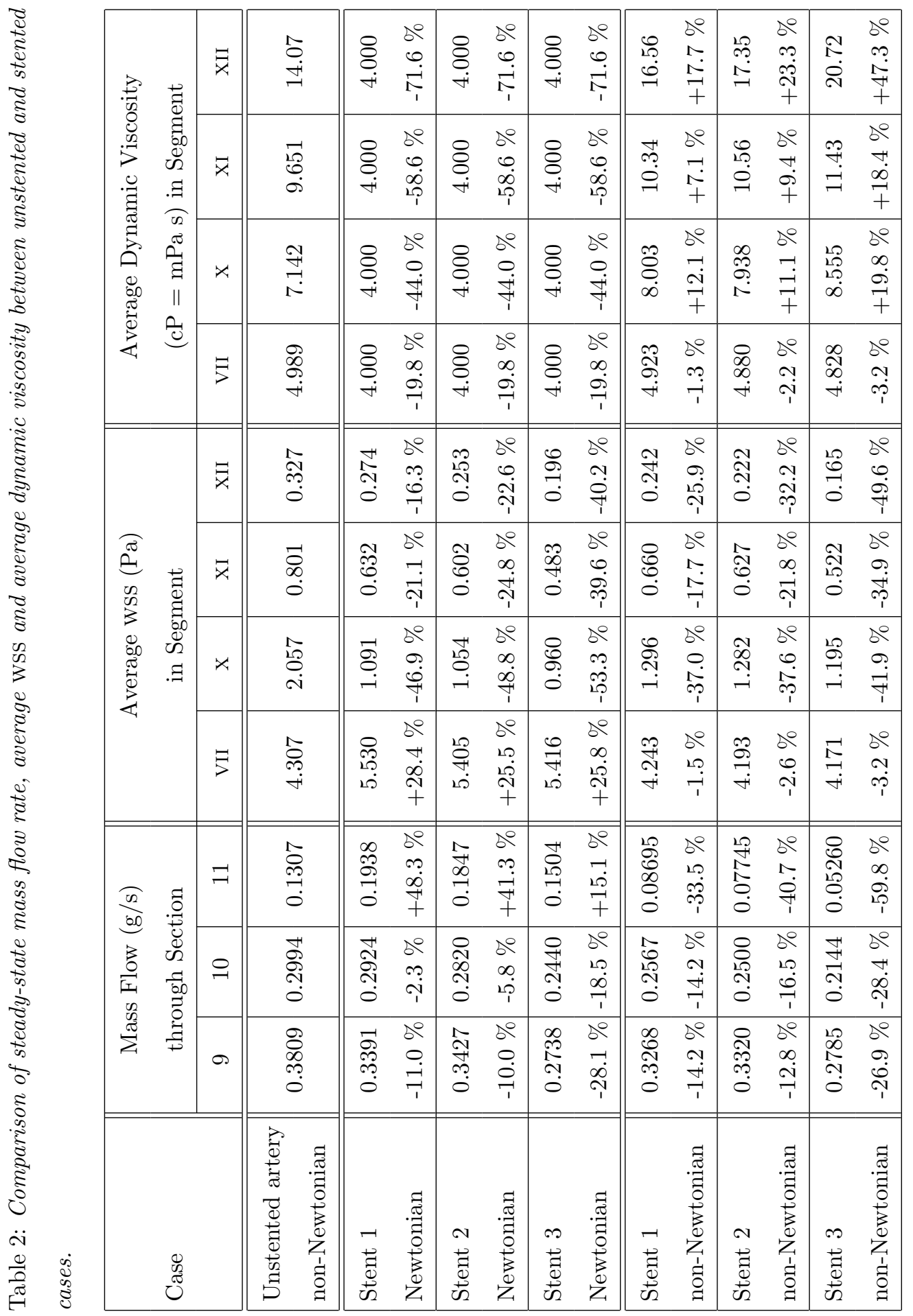


$9\left(0.3320 \frac{\mathrm{g}}{\mathrm{s}}\right.$ equal to a $12.8 \%$ reduction in the mass flow rate compared to the unstented case), but it drops more quickly than Stent 1 at Sections 10 and 11 . However, Stent 3 with a $26.9 \%$ reduction is much more effective in reducing the mass flow rate into the aneurysm (crossing Section 9), and so is expected to be a better clinical performer than the other two stents.

These results are in line with those of Radaelli et al. 2008 both in terms of flow rates (our Newtonian/non-Newtonian predictions are $2.4 \%$ above Radaelli's average) and flow rate reductions due to the stents (as shown in Table 3 below). In this respect we observe that the non-Newtonian character of blood has a small, but detectable, influence on stent performances, in particular for Stent 1 and Stent 2.

\subsubsection{Average wall shear stress}

Comparing the Wss in the unstented case with the three stented models (Figure 4 and Table 2), the use of a stent appears to be very effective in reducing the average WSS: reductions in segments X, XI and XII range between $18 \%$ and $38 \%$ for Stent 1 and Stent 2; and between $35 \%$ and $50 \%$ for Stent 3. In the context of steady-state modelling, Stent 3 is significantly and consistently better than Stent 1 and Stent 2. Referring to the table of results (Table 2) the average Wss in the Newtonian models is underestimated by $15-20 \%$ in the lower aneurysm area (Segment $\mathrm{x}$ ) where the fluid velocity is still quite high, and overestimated $13-19 \%$ in the upper aneurysm area (Segment XII) where the fluid velocity is low.

In the upper aneurysm area, the average wss is higher in the non- 


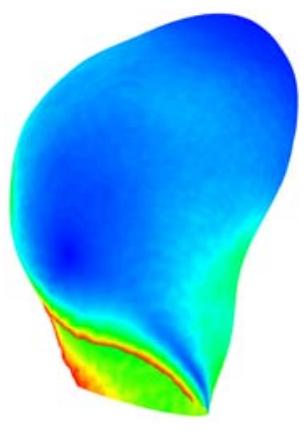

(a) Unstented

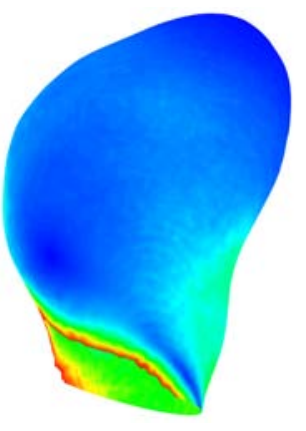

(b) Stent 1

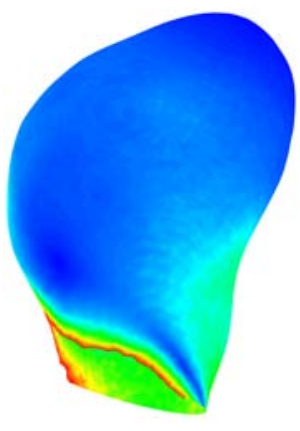

(c) Stent 2

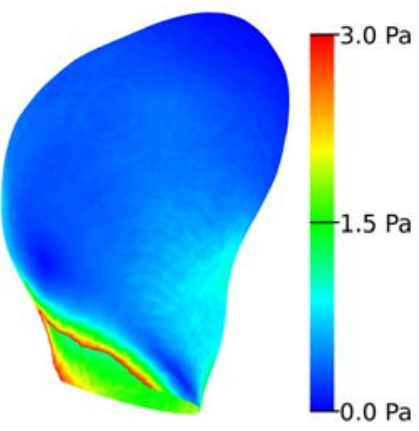

(d) Stent 3

Figure 4: WSS on surface of aneurysm for steady non-Newtonian simulations

Newtonian case as a result of the increase of the dynamic viscosity and the reduction of the mass flow rate in the aneurysm. This suggests that the Newtonian hypothesis might not be reliable for this application, in that errors in the prediction of wsS distribution can be significant even under steady-state conditions. Apart from this, data in Table 2 also reveal that wss average values are below the limit of $0.5 \mathrm{~Pa}$ in Segment XII (the aneurysm dome) for the unstented and all stented cases, independent of the rheological model adopted. However, in the non-Newtonian cases the peaks of wss in Segment XI are smoothed out. This significance has two features. Table 2 shows that the Newtonian to non-Newtonian effect is generally significant and, as importantly, position dependent.

These results demonstrate that Radaelli et al. 2008. were right to suggest the potential significance of this investigation and call into question the very recent remarks of Fu et al. 2010 "Studies using Newtonian fluid and nonNewtonian fluid on the same cerebral aneurysm models found that the main 
flow features are not greatly affected by the viscosity model".

\subsection{Pulsatile flow}

3.4.1. Mass flow rate over pulse cycle

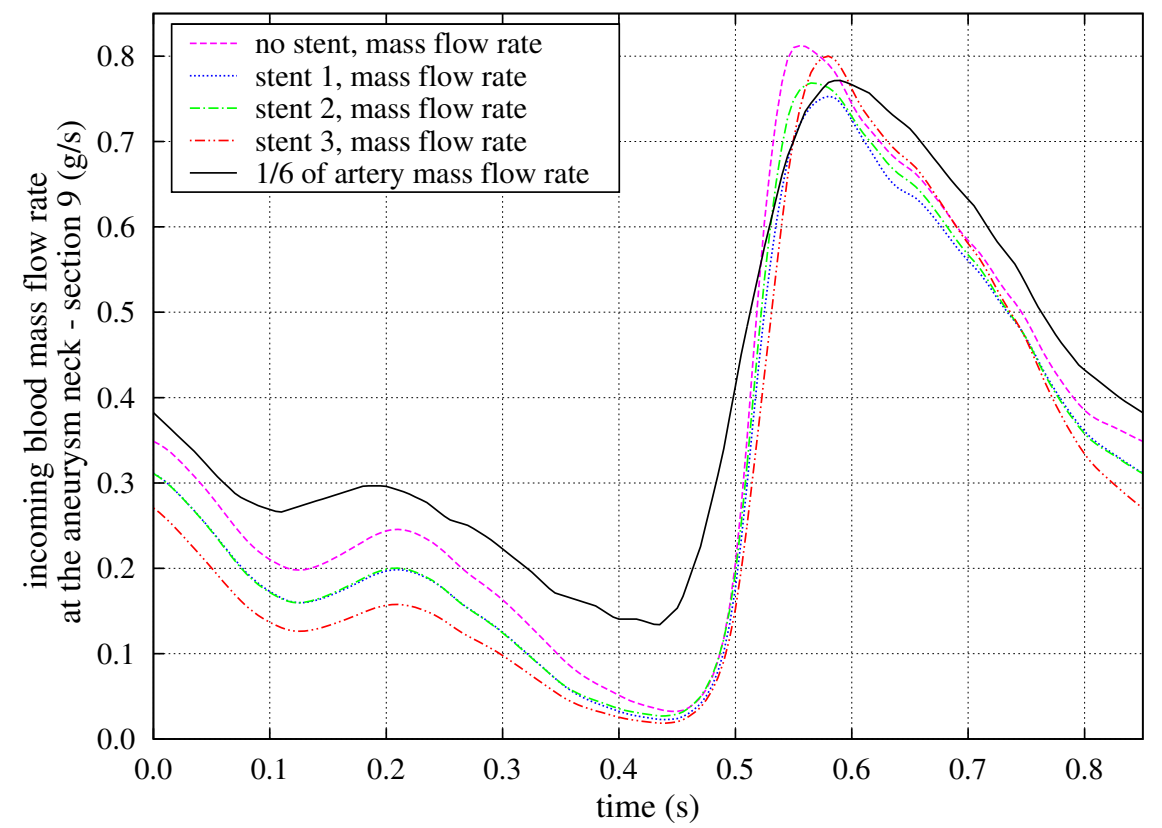

Figure 5: Mass flow through Section 9

After three pulse cycles the measured pressure difference in the aneurysm between beats was null and so the simulation was deemed to be truly periodic. The mass flow rate entering the aneurysm, i.e. crossing Section 9, is shown in Figure 5, together with the pulse cycle in the main artery, shown scaled to $1 / 6$-th of its amplitude. For the unstented case, the mass flow rate entering the aneurysm at time $0 \mathrm{~s}$ is equal to $15 \%\left(0.3488 \frac{\mathrm{g}}{\mathrm{s}}\right)$ of the mass flow rate in the main artery and remains in the range of $10 \%$ to $20 \%$ for all the cases 
investigated and over the pulse cycle, except at the lowest mass flow rate in the artery.

Stent 1 and Stent 2 reduce the mass flow rate over the entire cycle, whereas between 0.58 to 0.70 seconds Stent 3 either does not reduce or even increases the mass flow rate. This could have significance because it is around the peak mass flow rate in the cycle. There also appear to be phase differences around the peak for all the plots. VISC'07 operates around the time 0 s value not the peak at which Stent 3 is seen in Figure 5 to perform worse than Stent 1 and Stent 2.

Table 3 presents our results in terms of stent-dependent mass flow reductions compared with those of the constituent VISC'07 research groups and the overall average. Our comparative data are: (a) for Newtonian steadystate mass flow from Table 2, (b) for non-Newtonian steady-state mass flow from Table 2, (c) for non-Newtonian pulsatile mass flow, time-averaged over the cycle. Thus comparing (a) and (b) indicates, particularly for Stent 1 and Stent 2 values, the non-Newtonian effect, as discussed in section 3.3.1. Comparing (b) and (c) indicates the greater effect of pulsatility. It is pointed out that the Time-Averaged flow-rate in the main artery had been set equal to the VISC'07 datum for steady-state exercises; even so, pulsed-flow predictions give a time-averaged flow-rate value in the aneurysm $9 \%$ lower than Radaelli's average. Finally, the column-to-column stent comparisons indicate the general effect of stent design, which seems to be greater than the other two parameters. In particular, under pulsatile conditions all stent models still reduce the mass-flow in the saccular aneurysm, but their performance 
deteriorates slightly. In particular, the Time-Averaged percentage changes are less supportive of Stent 3 than other studies because of the poor performance around the peak mass flow rate cycle described above (Figure 5).

\subsubsection{Wall shear stress distribution over pulse cycle}

Looking at the wss cumulative distribution over the pulsatile cycle, Figure 6 indicates the extent to which each design of stent affects low wss (up to $0.5 \mathrm{~Pa}$ ). This cumulative distribution provides an immediate comparison between stent designs where the best design for an aneurysm is suggested to be the one that produces the higher curve below $0.5 \mathrm{~Pa}$ (i.e. greater area with low WsS).

In Table 4 the cumulative area wss below $0.5 \mathrm{~Pa}$ is averaged for 5 different times. Overall for Segment X, XI and XII in comparison to the unstented case, Stent 1 increases the area experiencing low wss by $59 \%$, Stent 2 by $60 \%$ and Stent 3 by $64 \%$.

\section{Discussion}

\subsection{Comparisons between models}

The results do not contradict Gijsen et al. (1999a,b), who, using both experiments and simulations, essentially state the importance of non-Newtonian (shear-thinning) blood modelling since it alters significantly the velocity profiles. As a non-Newtonian model produces higher fluid viscosities in the aneurysm region (where the shear rate is low) compared to the Newtonian model, this implies that the fluid tends to reduce its speed much more quickly 


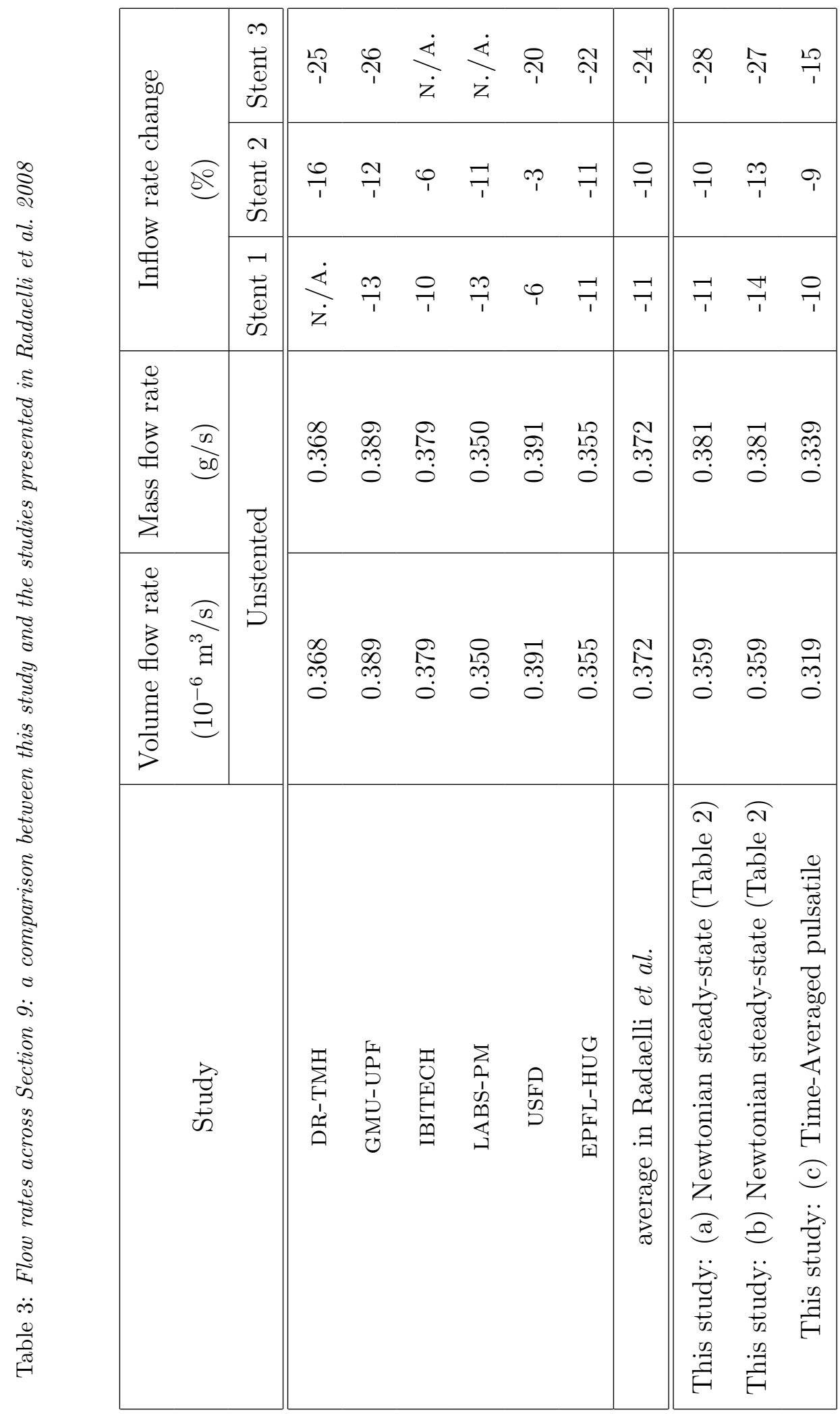




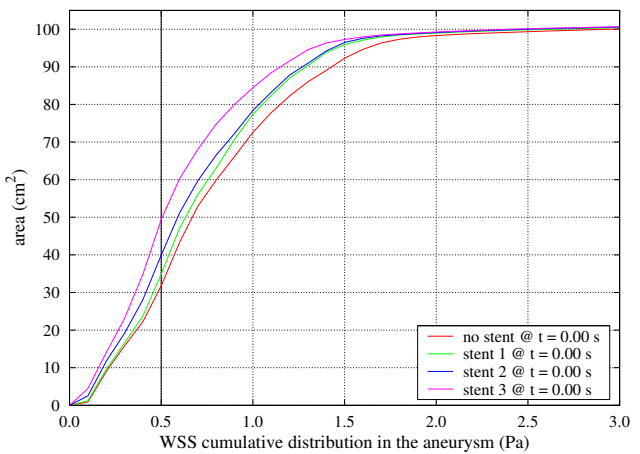

(a) time $0.00 \mathrm{~s}$

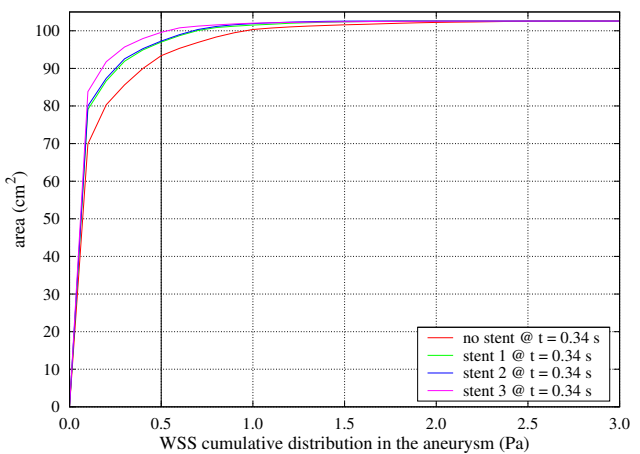

(c) time $0.34 \mathrm{~s}$

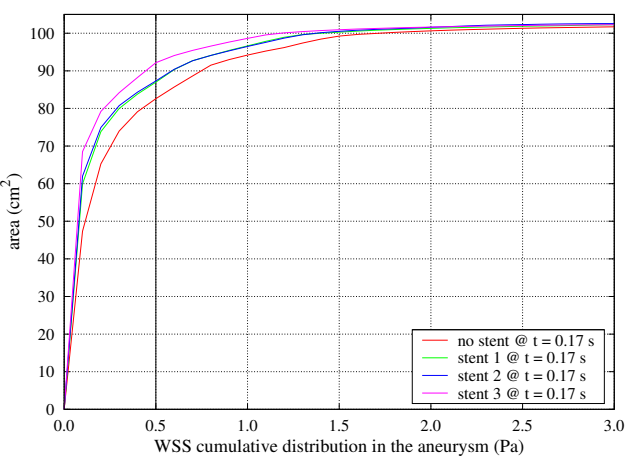

(b) time $0.17 \mathrm{~s}$

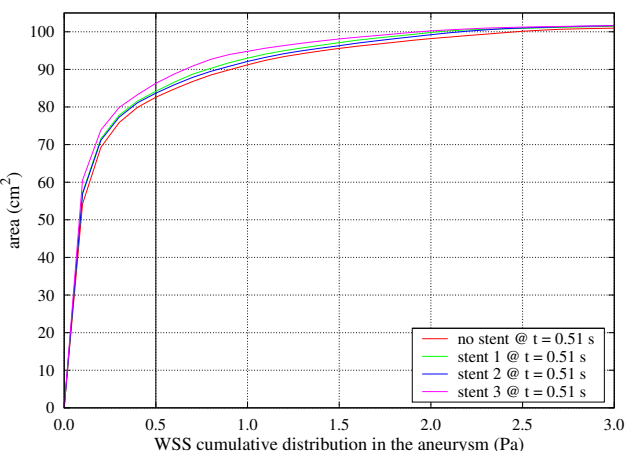

(d) time $0.51 \mathrm{~s}$

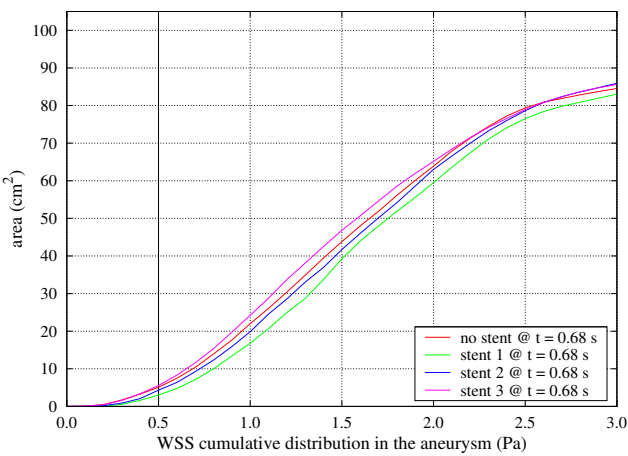

(e) time $0.68 \mathrm{~s}$

Figure 6: Cumulative area distribution of wss over pulsatile cycle 


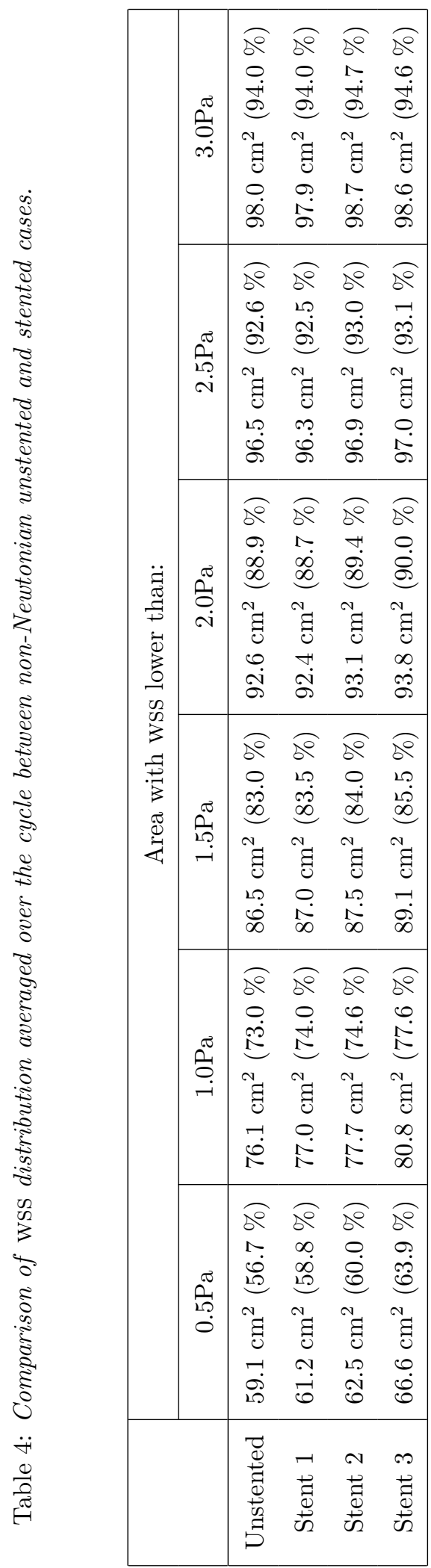


inside the aneurysm, and hence the non-Newtonian model produces a smaller mass flow rate. Also, the lower velocity gradient promotes a lower wss while a higher viscosity promotes a higher wss.

From Table 2, it seems that the average values of the Wss in very low shear areas (upper aneurysm - Segment XII) obtained with the non-Newtonian model are due to a balancing of the two effects of reduced velocity and increased viscosity, which are much the same for Newtonian and non-Newtonian cases. In the lower aneurysm however, the effect of the increased viscosity is dominant and the Newtonian model significantly underestimates the average wss. In the main artery, the models were set to have approximately the same average velocity and viscosity however the Newtonian assumption significantly overestimates the wss by between $28.9 \%$ and $30.3 \%$. This must be due to a smoother velocity profile with lower gradients at the wall that is produced by the non-Newtonian assumption. Additionally, the max wss is overestimated with the Newtonian model. Overall, it seems that the nonNewtonian hypothesis redistributes the velocity profiles and the wss in a more uniform and smooth way and thus peaks are smoothed out (Figure 7). However, as the shear rate decreases the viscosity grows quickly, while the mass flow rate decreases slowly at first and then increasingly faster. This means that, despite the Newtonian hypothesis overestimating the wss in the artery, it underestimates it in the aneurysm, except in those areas where the WSS is extremely low.

Computing Reynolds and Womersley numbers it transpires, based on the average artery dimension, on the average fluid velocity and on the average 


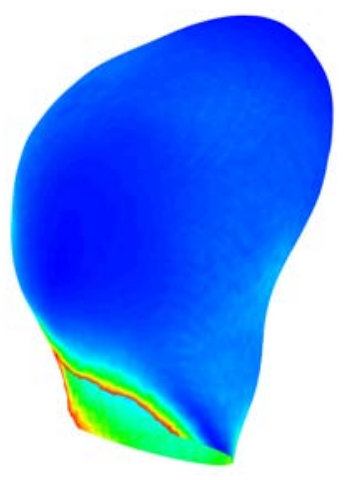

(a) A

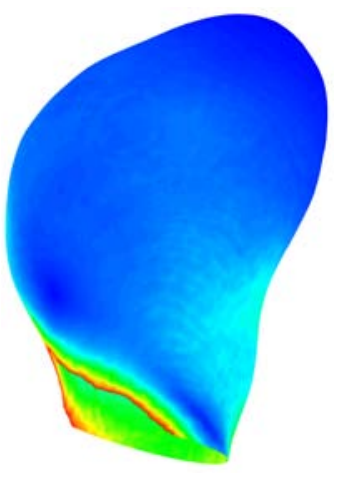

(b) B

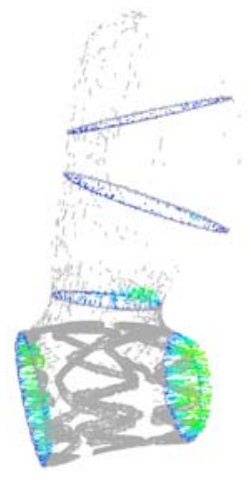

(c) $\mathrm{C}$

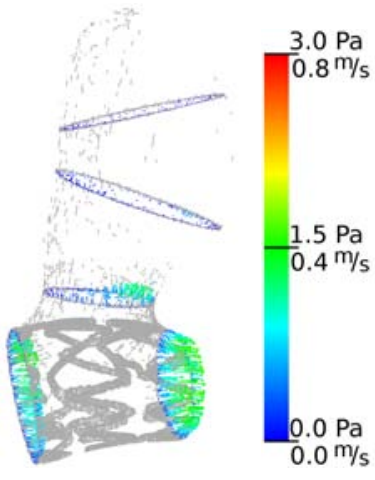

(d) D

Figure 7: WSS and velocity vectors in aneurysm region for stent 3 steady simulation: Newtonian versus non-Newtonian: (a) WSS, Newtonian case; (b) Wss, non-Newtonian case; (c) velocity vectors, Newtonian case; (d) velocity vectors, non-Newtonian case

blood dynamic viscosity in the artery that $\mathrm{Re}=180$, and Wo $=4.7$. From literature it is well-known that for Wo $<1$ the effects of inertia are insignificant and there is no difference between a quasi-static analysis and a non-stationary analysis, that is, pressure and flow are perfectly in phase. For Wo $>10$ the effects of inertia cannot be ignored. As Wo $=4.7$ is in between these regions, we can accept, to a first approximation, the assumption of ignoring pulsatility but we have found that including pulsatility gives an important result in that there is a significant region around the maximum cyclic mass flow rate where only Stent 1 and Stent 2 reduce the mass flow rate. VISC'07 operates around the time $0 \mathrm{~s}$ value of the pulsatile cycle rather than the peak, which is where Stent 3 is seen in Figure 5 to perform worse than Stent 1 and Stent 2. This result justifies the incorporation of pulsatility and confirms the indication of its possible importance by Radaelli et al. 2008 
In addition, there is the very recent comment of $\mathrm{Fu}$ et al. 2010 who don't include pulsatility because it would cost too much, "Since the number of elements in the volume mesh is millions in this study, pulsatile flow simulations would require several weeks of computational time for each model, and the six models need to be compared. So the steady-state flow simulations in this work were carried out to keep the CPU times reasonable." The computational cost is indeed very high but we have seen that the changes over the cycle are not insignificant (10\% to $20 \%$ of the arterial mass flow rate). While these tend to average out over the cycle, we show that over an important part of that cycle only Stent 1 and Stent 2 reduce the mass flow rate. Overall, if time and computational sources allow it, a pulsatile model gives valuable additional information.

\subsection{Implications for stent design}

The method presented for performing CFD simulations and post-processing of a stented cerebral artery seems to be reliable both in view of the mesh sensitivity analysis performed for testing the consistency of the results, and in light of the good agreement with the results of VISC'07 (Radaelli et al. 2008) under steady-state conditions. The present "complete" (i.e. pulsatile and non-Newtonian) analysis however shows that a stent geometry "best choice" based on "simplified" conditions (steady and Newtonian) might contain some uncertainty, since in fact Stent 3 (identified as the best in VISC'07 studies) is found to produce an increase in the flow-rate entering the aneurysm. This does not necessarily mean that the stent choice itself is compromised, but 
could provide additional information to the clinician to be considered in the stent selection. In more general terms, the moderate (or even negative) effect of the stents used in VISC'07 at the cycle peak could prompt a revision in the testing conditions and, eventually, in stent design. A possible suggestion of practical concern, in view of the present results, is to extend the numerical testing of a stent at steady-state conditions to include predictions at the cycle-peak flow-rate conditions other than the cycle-averaged flow-rate value, as used in VISC'07. Finally, the cumulative distribution performance measures presented are intuitive and effective and could be used in future work for assessing the performance of a stent design for a given patient.

\subsection{Future work}

The effect of the thin stent wires on local flow and the influence of inflow angle to the stented zone are of interest:

- Local flow around wires because this is where low oscillatory shear flows are associated with disease.

- Inflow angle, which depends mainly on the curvature of the main artery and the location of the aneurysm sac, because this represents a noise factor between patients that impinges differently upon a given stent design and affects Wss distribution. Friedman et al. (1996) propose the planarity of the aortic bifurcation to be a geometric risk factor since it can vary widely from individual to individual, diversely affecting the blood flow, which means that it can play a role in predispositions to 
atherosclerosis. Aneurysm inflow depends partly on the curvature of the parent vessel and an inflow angle of 45 degrees has been found to produce the highest risk of aneurysm rupture Liou and Li (2008). Inflow characteristics more generally are affected by stent position, which will not be the same for all patients.

Uncertainties in surgical procedures include those associated with variations in stent position, artery geometry and flow characteristics. A stent design must generally function despite such uncertainty. Radaelli et al. (2008), state "due to the fact that current stent designs are not optimized to act as flow diverters and their performance may depend on a correct positioning during intervention. Novel stent designs should aim at providing the same flow diversion irrespective of the actual positioning before deployment." In other words, the stent design should be more robust. The variation in arterial haemodynamics due to anatomical and other geometric variations call for modelling to be patient-specific but apart from nominal geometry effects Nguyen et al. (2008), little attempt has yet been made to quantify the effect of variability in arterial geometry or stent placement on wss. A simple approach has been proposed by the authors in Atherton and Collins (2006) and Cavazzuti et al. (2008). Geometric variations call for patient-specific modelling but a patient-specific stent design will still be subject to uncertainties in location and flow characteristics etc. Thus we propose that more robust stent designs are required that are able to better accommodate such uncertainties.

Design optimisation methods have developed to address variability, for 
example Robust Engineering Design (RED) whose significance is in its aim to render the design insensitive to uncertainty (Atherton et al. (2002)). The variation in wsS over the aneurysm and artery walls that is caused by geometric and flow uncertainties, as already mentioned, can form the basis of a performance measure for comparing the effects of different stent designs, stent position and other geometric risk factors.

\subsection{Conclusions}

In this paper a set of VISC'07 predictions have been reported that take into account non-Newtonian and pulsatile effects, as acknowledged by Radaelli et al. (2008), as of potential significance. The resulting Newtonian to nonNewtonian comparison shows that the effect is quantitatively significant and, moreover, position dependent. The extra computational demand of the nonNewtonian model is insignificant and we therefore conclude that there is little justification for assuming Newtonian viscosity.

On the other hand, the pulsatility predictions were highly computationally intensive and, as in our discussion of Figure 5, the relative reduction of mass flow rate is not the same over the whole cycle. To the extent that VISC'07 challenge was meant to be able to discriminate between three stent designs, our results consistently agree. However, we have found a significant quantitative effect because of Stent 3 operating in a counter beneficial way for a substantial fraction of the pulsatile cycle. Hence there is added understanding of stent performance in incorporating the flow pulsatility. The spirit of these conclusions is consistent with the fact that the Womersley value of 
4.7 lies midway between the criteria for allowing steady-state $($ Wo $<1$ ) and for requiring a pulsatile model (Wo $>10$ ). Using wss distribution as the basis of a performance measure is meaningful. In this study, quantifying the distribution below a target wSS value $(0.5 \mathrm{~Pa})$ was a good discriminator between stent designs, and stent 3 emerges as the best design, which is consistent with the results published in the literature.

\section{Conflict of interest statement}

The authors have no financial or personal relationships with other people or organisations that could inappropriately influence (bias) their work.

\section{Acknowledgements}

We thank Dr Matthieu De Beule from the Institute Biomedical Technology, Ghent University, Belgium for his generous advice on how to implement the Magics software. Also, our thanks to Dr Peter Flynn, Consultant Radiologist, Victoria Hospital, Belfast Trust, for his helpful comments on clinical aspects of the paper.

\section{References}

Atherton, M.A., Tesch, K., Collins, M.W., 2002. Effects of stents under asymmetric inflow conditions. Biorheology 39(3-4), 501-506.

Atherton, M.A., Collins, M.W., 2006. Vascular stents: performance measures for robust design development. Abstracts of Third Physiological 
Flow Network meeting, University of Oxford, 18-19 April 2006, 23. http://www2.imperial.ac.uk/ssherw/physflow/pfn/Meetings/Oxford/Programme.pdf (Accessed 07 July 2010)

Balossino, R., Gervaso, F., Migliavacca, F., Dubini, G., 2008. Effects of different stent designs on local hemodynamics in stented arteries. Journal of Biomechanics 41 (5), 1053-1061.

Boussel, L., Rayz, V., McCulloch, C., Matin, A., Acevedo-Bolton, G., Lawton, M., Higashida, R., Smith, W.S., Young, W.L., Saloner, D., 2008. Aneurysm growth occurs at region of low wall shear stress. Stroke, 39, 2997-3002.

Caro, C.G., Fitz-Gerald, J.M., Schroter, R.C., 1971. Atheroma and arterial wall shear. Proceedings of the Royal Society of London, Series B, 177 (46), 109-159.

Cavazzuti, M., Atherton, M.A., Barozzi, G.S., Collins, M.W., 2008. Cerebral stents: A performance measure of wall shear stress for robustness. Bioengineering08 Conference, Imperial College, 18-19 September 2008, s2-P-003. http://bioeng.fontismedia.com/general/programme.pdf (Accessed 07 July 2010)

Cavazzuti, M., Atherton, M.A., Collins, M.W., Barozzi, G.S., 2010. Beyond the Virtual Intracranial Stenting Challenge 2007: non-Newtonian and flow pulsatility effects. Journal of Biomechanics 43 (13), 2645-2647. 
Cebral, J.R., Castro, M.A., Appanaboyina, S., Putman, C.M., Millan, R.D., Frangi, A.F., 2005. Efficient pipeline for image-based patient-specific analysis of cerebral aneurysm haemodynamics: technique and sensitivity. IEEE Transactions on Medical Imaging 24 (4), 457-467

Chen, J., Lu, X-Y., Wang, W., 2006, Non-Newtonian effects of blood flow on hemodynamics in distal vascular graft anastomoses. Journal of Biomechanics 39 (11), 1983-1995.

Fisher, C. and Rossmann, J.S., 2009. Effect of non-Newtonian behavior on hemodynamics of cerebral aneurysms, Journal of Biomechanical Engineering,131(9): 091004.1-9.

Fluent 6.3 User Guide, 2006, section 8.4.5.

Friedman, M.H., Baker, P.B., Ding, Z, Kuban, B.D., 1996. Relationship between the geometry and quantitative morphology of the left anterior descending coronary artery. Atherosclerosis 125, 183-192.

Fu, W., Gu, Z., Meng, X., Chu, B., Qiao, A., 2010. Numerical simulation of hemodynamics in stented intenral carotid aneurysm based on patientspecific model. Journal of Biomechanics 43 (7), 1337-1342.

Gambaruto, A.M., Peiro, J., Doorly, D.J., Radaelli, A.G., 2008. Reconstruction of shape and its effects on flow in arterial conduits. International Journal for Numerical Methods in Fluids, 57 (5), 495-517. 
Gijsen, F.J.H., van de Vosse, F.N., Janssen, J.D., 1999. The influence of the non-Newtonian properties of blood on the flow in larger arteries: steady flow in a carotid bifurcation model. Journal of Biomechanics 32 (6), $601-608$.

Gijsen, F.J.H., Allanic, E., van de Vosse, F.N., Janssen, J.D., 1999. The influence of the non-Newtonian properties of blood on the flow in larger arteries: unsteady flow in a 903 curved tube. Journal of Biomechanics $32(6), 705-713$.

Ishikawa, T., Guimaraes, L.F.R., Oshima, S., Yamane, R., 1998. Effect of non-Newtonian property of blood on flow through stenosed tube. Fluid Dynamics Research 22, 251-264.

Johnson, B.M., Johnson, P.R., Corney, S., Kilpatrick, D., 2004. NonNewtonian blood flow in human right coronary arteries: steady state simulations. Journal of Biomechanics 37 (5), 709-713.

Karmonik, C., Klucznik, R., Haykal, H., Benndorf, G., 2008. Understanding the dangers of aneurysms. ANSYS ADVANTAGE II (2), 38-39.

Kim, M., Taulbee, D.B., Tremmel, M., Meng, H., 2008. Comparison of two stents in modifying cerebral aneurysm hemodynamics. Annals of Biomedical Engineering 36 (5), 726-741.

Lee, S.-W., Steinman, D.A., 2006. On the relative importance of rheology for image-based CFD models of the carotid bifurcation. Journal of Biomechanics 39 (1), S283. 
Liou, T-M., Li, Y-C., 2008. Effects of stent porosity on hemodynamics in a sidewall aneurysm model. Journal of Biomechanics 41 (5), 1174-1183.

Nguyen, K.T., Clark, C.D., Chancellor, T.J., Papvassiliou, D.V., 2008. Carotid geometry effects on blood flow and on risk for vascular disease. Journal of Biomechanics 41 (1), 11-19.

Owen, I., Gray, J., Escudier, M., Poole, R., 2005. The importance of the nonNewtonian characteristics of blood in flow modelling studies. Abstracts of Second Physiological Flow Network meeting, University of Edinburgh, 26-27 September 2005.

Perktold, K., Peter, R., Resch, M., 1989. Pulsatile non-Newtonian blood flow simulation through a bifurcation with an aneurysm. Biorheology 26 (6), 1011-1030.

Punchard, M.A., Stenson-Cox, C., O'Cearbhaill, E.D., Lyons, E., Gundy, S., Murphy, L., Pandit, A., McHugh, P.E., Barron, V., 2007. Endothelial cell response to biomechanical forces under simulated vascular loading conditions. Journal of Biomechanics 40 (14), 3146-3154.

Radaelli, A.G., Augsburger, L., Cebral, J.R., Ohta, M., Rufenacht, D.A., Balossino, R., Benndorf, G., Hose, D.R., Marzo, A., Metcalfe, R., Mortier, P., Mut, F., Reymond, P., Socci, L., Verhegghe, B., Frangi, A.F., 2008. Reproducibility of haemodynamical simulations in a subjectspecific stented aneurysm model - A report on the Virtual Intracranial Stenting Challenge 2007. Journal of Biomechanics 41 (10), 2069-2081. 
Shibeshi, S.S., Collins, W.E., 2005. The Rheology of Blood Flow in a Branched Arterial System. Applied Rheology 15 (6), 398-405.

Shimogonya, Y., Ishikawa, T., Imai, Y., Matsuki, N., Yamaguchi, T., 2009. Can temporal fluctuation in spatial wall shear stress gradient initiate a cerebral aneurysm? A proposed novel hemodynamic index,the gradient oscillatory number(GON). Journal of Biomechanics 42 (4), 550-554.

Steinman, D.A., Milner, J.S., Norley, C.J., Lownie, S.P., Holdsworth, D.W., 2003. Image-based computational simulation of flow dynamics in a giant intracanial aneurysm. American Journal of Neuroradiology 24 (4), 559566.

Utter, B., Rossman, J.S., 2007. Numerical simulation of saccular aneurysm hemodynamics: Influence of morphology on rupture risk. Journal of Biomechanics 40 (12), 2716-2722.

Valencia, A.A., Guzman, A.M., Finol, E.A., Amon, C.H., 2006. Blood flow dynamics in saccular aneurysm models of the basilar artery. Journal of Biomechanical Engineering 128 (4), 516-526.

VISC 2007 The 1st Virtual Intracranial Stenting Challenge http://www.cilab.upf.edu/visc06 (accessed 29 January 2009)

\section{List of Figures}

$1 \quad$ Unstented aneurysm and the three VISC'07 stent models . . . . 7 
2 Sections of aneurysm model for post processing . . . . . . . . . 11

3 Velocity vectors show where flow impinges on aneurysm neck through Stent 3 . . . . . . . . . . . . . . 13

$4 \quad$ WSS on surface of aneurysm for steady non-Newtonian simu-

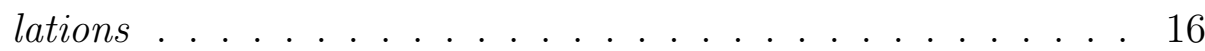

5 Mass flow through Section 9 . . . . . . . . . . . . . . 17

$6 \quad$ Cumulative area distribution of wss over pulsatile cycle . . . 21

$7 \quad$ WSS and velocity vectors in aneurysm region for stent 3 steady simulation: Newtonian versus non-Newtonian: (a) Wss, Newtonian case; (b) WSS, non-Newtonian case; (c) velocity vectors, Newtonian case; (d) velocity vectors, non-Newtonian case 24

\section{List of Tables}

1 Mesh sensitivity comparison based on mass flow through Section $9 \ldots \ldots \ldots \ldots \ldots \ldots$

2 Comparison of steady-state mass flow rate, average wss and average dynamic viscosity between unstented and stented cases. 14

3 Flow rates across Section 9: a comparison between this study and the studies presented in Radaelli et al. 2008 . . . . . . . . 20

4 Comparison of Wss distribution averaged over the cycle between non-Newtonian unstented and stented cases. . . . . . . . 22 